\title{
Journal of Ultrasound indexed in MEDLINE
}

\author{
Ferdinando Draghi ${ }^{1}$
}

Published online: 1 April 2016

(C) Società Italiana di Ultrasonologia in Medicina e Biologia (SIUMB) 2016

The National Library of Medicine has just accepted the Journal of Ultrasound (JUS) for listing and indexing in MEDLINE. Articles from JUS will begin appearing in MEDLINE/PubMed/Index Medicus from volume 19 (first issue 2016).

It is a great pleasure for me as Editor-in-Chief and for the Editorial Board to announce this good news to all members of SIUMB and to all Readers.

The Journal was founded in 1990 as a tool for continuing medical education of the Italian Society for Ultrasound in Medicine and Biology (SIUMB), which is a multidisciplinary society. Right from the beginning, the objective was first of all to support theoretical and practical training of ultrasound specialists. With this purpose in mind, the contents of the issues are carefully selected in collaboration with international scientific societies and recognized key opinion leaders.

The contents of the Journal reflect the multidisciplinary nature of the scientific society as also articles dealing with ultrasound physics and the characteristics of ultrasound contrast agents are published in addition to studies involving extra-medical topics and veterinary ultrasound.

The Editorial Board consists of internationally recognized key opinion leaders residing and working in various parts of the world with first-hand experience in research and scientific publication.

Ferdinando Draghi

f.draghi@smatteo.pv.it

1 IRCCS, Policlinico San Matteo, Istituto di Radiologia, Pavia, Italy
Over the last 6 years, the readership has become increasingly international. In 2013, $66 \%$ of the readers were European; in 2014, the percentage of European readers was reduced to $51 \%$ while Asia-Pacific Region readers increased from 14 to $20 \%$ and North American readers from 11 to $18 \%$.

In addition, the number of submissions has increased. In 2013 the articles submitted were 32 and in 2014 the journal had 61 manuscripts submitted, an improvement which further evidences the increasing internationalization of the journal. Also the full text article downloads had good growth: in 2013 were 2.367 and in 2014 reached 6.220, data which proves the improvement of the quality of the contents. These are the results of a meticulous review process which is aimed towards accepting manuscripts of a high scientific standard to provide a valuable contribution to the knowledge of the disciplines.

Indexing in MEDLINE is an important step for JUS in becoming an internationally known journal of sonography. Our next big concern is, of course, to obtain an impact factor.

On behalf of the Editorial Board, I earnestly solicit the cooperation of all SIUMB members and readers for the further development of JUS. 